\title{
Cmah deficiency may lead to age-related hearing loss by influencing miRNA-PPAR mediated signaling pathway
}

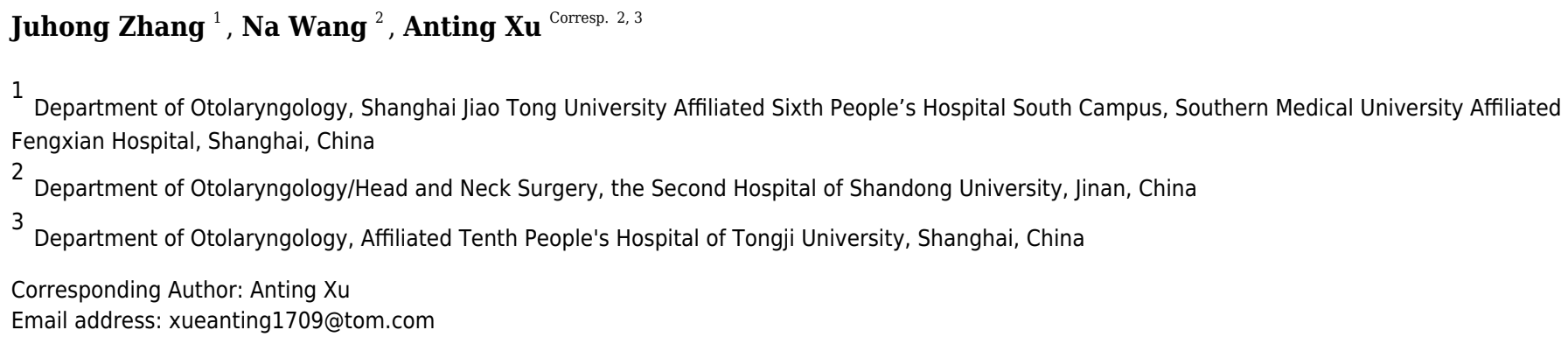

Background: Previous evidence has indicated CMP-Neu5Ac hydroxylase (Cmah) disruption inducesaging-related hearing loss ( $A H L)$. However, its function mechanisms remain unclear. This study was to explore the mechanisms of AHL by using microarray analysis in the Cmah deficiency animal model. Methods: Microarray dataset GSE70659 was available from the Gene Expression Omnibus database, including cochlear tissues from wild-type and Cmah-null C57BL/6J mice with old age (12 months, $n=3$ ). Differentially expressed genes (DEGs) were identified using the Linear Models for Microarray data method and protein-protein interaction (PPI) network was constructed using data from the Search Tool for the Retrieval of Interacting Genes database followed by module analysis. Kyoto encyclopedia of genes and genomes pathway enrichment analysis was performed using The Database for Annotation, Visualization and Integrated Discovery. The upstream miRNAs and potential small-molecule drugs were predicted by miRwalk2.0 and Connectivity Map, respectively. Results: A total of 799 DEGs (449 upregulated and 350 downregulated) were identified. Upregulated DEGs were involved in Cell adhesion molecules (ICAM1, intercellular adhesion molecule 1) and tumor necrosis factor (TNF) signaling pathway (FOS, FBJ osteosarcoma oncogene; ICAM1), while downregulated DEGs participated in PPAR signaling pathway (PPARG, peroxisome proliferator-activated receptor gamma). A PPI network was constructed, in which FOS, ICAM1 and PPARG were ranked as hub genes and PPARG was a transcription factor to regulate other target genes (ICAM1, FOS). Function analysis of two significant modules further demonstrated PPAR signaling pathway was especially important. Furthermore, mmu-miR-130b-3p, mmu-miR-27a-3p, mmu-miR-27b-3p and mmu-miR-721 were predicted to regulate PPARG. Topiramate were speculated to be a potential small-molecule drug to reverse DEGs in AHL. Conclusions: PPAR mediated signaling pathway may be an important mechanism for AHL. 
strategies for ALH by upregulating PPARG. 
1 Cmah deficiency may lead to age-related hearing loss by influencing miRNA-PPAR

2 mediated signaling pathway

3 Running title: miRNA-PPAR for AHL

4 Juhong Zhang ${ }^{1}$, Na Wang ${ }^{2}$, Anting $\mathrm{Xu}^{2,3 \#}$

5 'Department of Otolaryngology, Shanghai Jiao Tong University Affiliated Sixth People's

6 Hospital South Campus, Southern Medical University Affiliated Fengxian Hospital, Shanghai, 7 201411, China;

$8{ }^{2}$ Department of Otolaryngology/Head and Neck Surgery, the Second Hospital of Shandong 9 University, Jinan, 250033, China;

$10{ }^{3}$ Department of Otolaryngology, Affiliated Tenth People's Hospital of Tongji University, 11 Shanghai, 200072, China.

13 Corresponding author: Xu Anting.

14 Email: xueanting1709@tom.com; Telephone: +86-0531-85875317; Fax: +86-0531-85875458.

15 Corresponding address: Department of Otolaryngology/Head and Neck Surgery, the Second 16 Hospital of Shandong University, Beiyuan Street 247, Tianqiao District, Jinan, 250033, China. 
22 Background: Previous evidence has indicated CMP-Neu5Ac hydroxylase (Cmah) disruption

23 induces aging-related hearing loss (AHL). However, its function mechanisms remain unclear.

24 This study was to explore the mechanisms of AHL by using microarray analysis in the Cmah

25 deficiency animal model.

26 Methods: Microarray dataset GSE70659 was available from the Gene Expression Omnibus

27 database, including cochlear tissues from wild-type and Cmah-null C57BL/6J mice with old age

28 (12 months, $\mathrm{n}=3$ ). Differentially expressed genes (DEGs) were identified using the Linear

29 Models for Microarray data method and protein-protein interaction (PPI) network was constructed using data from the Search Tool for the Retrieval of Interacting Genes database followed by module analysis. Kyoto encyclopedia of genes and genomes pathway enrichment analysis was performed using The Database for Annotation, Visualization and Integrated Discovery. The upstream miRNAs and potential small-molecule drugs were predicted by miRwalk2.0 and Connectivity Map, respectively.

Results: A total of 799 DEGs (449 upregulated and 350 downregulated) were identified. Upregulated DEGs were involved in Cell adhesion molecules (ICAM1, intercellular adhesion molecule 1) and tumor necrosis factor (TNF) signaling pathway (FOS, FBJ osteosarcoma oncogene; ICAM1), while downregulated DEGs participated in PPAR signaling pathway (PPARG, peroxisome proliferator-activated receptor gamma). A PPI network was constructed, in which FOS, ICAM1 and PPARG were ranked as hub genes and PPARG was a transcription factor to regulate other target genes (ICAM1, FOS). Function analysis of two significant modules further demonstrated PPAR signaling pathway was especially important. Furthermore, 
43 mmu-miR-130b-3p, mmu-miR-27a-3p, mmu-miR-27b-3p and mmu-miR-721 were predicted to 44 regulate PPARG. Topiramate were speculated to be a potential small-molecule drug to reverse 45 DEGs in AHL.

46 Conclusions: PPAR mediated signaling pathway may be an important mechanism for AHL.

47 Downregulation of the above miRNAs and use of topiramate may be potential treatment 48 strategies for ALH by upregulating PPARG.

49

50

51

52

53

54

55

56

57

58

59

60

61

62

63

Introduction

Peer] reviewing PDF | (2019:01:34403:1:1:NEW 18 Mar 2019) 
64 Hearing loss is the most common sensorineural deficit in the elderly and it is estimated that

65700 million persons have moderate to profound hearing loss worldwide in 2015, with

66

67

68

69

approximately $30 \%$ of them occurred in their seventies and $50 \%$ in their eighties (Niklaus et al. 2011; Hjalte et al. 2012; Quaranta et al. 2015). Age-related hearing loss (AHL) can lead to communication difficulties and cause social isolation, depression and anxiety, all of which severely influence the quality of life of patients (Ciorba et al. 2012). Furthermore, AHL is demonstrated to trigger cognitive function impairment in patients and thus may impose a large economic burden on families and society (Peelle \& Wingfield 2016). Thus, how to manage AHL has been an important public health issue.

Increasing evidence has indicated that oxidative stress is a crucial pathogenesis for AHL (Fujimoto \& Yamasoba 2014). Plasma reactive oxygen species (ROS) levels (i.e. hydrogen peroxide, hypochlorite and hydroxyl radicals) are observed to be significantly elevated (Hwang et al. 2012), while antioxidant retinol and zinc levels are significantly reduced in AHL patients (Lasisi \& Lasisi 2015). Linear regression reveals ROS and radical scavenger levels are positively and negatively associated with hearing thresholds of patients, respectively (Hwang et al. 2012; Lasis \& Fehintola 2011). Furthermore, AHL animal model experiments also confirmed ROS excessively accumulated (Riva et al. 2007), but antioxidant enzymes [such as superoxide dismutase (SOD), reduced glutathione (GSH)/oxidized glutathione (GSSG)] strongly decreased in the cochlear spiral ganglion neurons and hair cells (Coling et al. 2009; Menardo et al. 2012). Even, AHL phenotype can be directly mimicked by selective knockout of SOD1 gene in mice (Watanabe et al. 2014). Oxidative stress may result in mitochondrial DNA mutations (Markaryan 
et al. 2009; Yamasoba et al. 2007) and subsequently initiate BCl-2/Bax and caspase-3 mediated apoptotic pathways in the sensory cells and neurons of the cochlea (Du et al. 2015; Huang et al. 2016), which ultimately contribute to the development of hearing loss. Accordingly, supplementation of antioxidants (i.e. vitamin C, N-acetyl-cysteine) (Ding et al. 2016; Kang et al. 2014) or suppression of cell apoptosis of (i.e. caloric restriction, Erlong Zuoci decoction) (Dong et al. 2016; Someya et al. 2010a) may be underlying strategies to delay the onset of AHL and prevent pathological damages in the cochlea. However, the mechanisms of AHL remain not completely understood and current preventative or therapeutic interventions have not been universally acknowledged. Thereby, there is still a need to investigate the etiology of AHL to develop potential approaches for intervention of AHL.

CMP-Neu5Ac hydroxylase (Cmah) is an enzyme to catalyze the hydroxylation of Nacetylneuraminic acid (Neu5Ac) to N-glycoloylneuraminic acid (Neu5Gc). Neu5Gc is an important sialic acid and thus may play an important role for maintaining the structural and function of auditory system (Go et al 2011; Inokuchi et al. 2017). Cmah-deficient mice are shown to exhibit reduced hearing sensitivity in old age, accompanied with loss of sensory hair cells, spiral ganglion neurons, and/or stria vascularis degeneration throughout the cochlea (Kwon et al. 2015; Hedlund et al. 2007). These studies indicate mice with Cmah-null can act as a model for studying the mechanisms of AHL in humans, which had been used in the study of Kwon et al. (Kwon et al. 2015). Based on a high throughput microarray analysis technology, Kwon et al. demonstrated there were 631 up-regulated and 729 down-regulated genes in Cmah-null micederived cochlear tissues compared to control mice-derived cochlear tissues. Function enrichment 
106 analysis and PCR validation suggsted downregulated sirtuin deacetylase 3 (Sirt3), a 107 mitochondrial NAD+-dependent deacetylase, may be involved in AHL via decreasing the expression of Fox1 and then promoting the production of ROS (Kwon et al. 2015). The key roles of Sirt3 for the development of AHL were also proved in the studies of other scholars (Someya et al. 2010b; Zeng et al. 2014). Therefore, manipulation of Sirt3 expression might represent a new approach to combat AHL. However, the use of Cmah-null mice to investigate the mechanism of AHL remains rarely reported.

The present study aimed to screen more crucial genes for explaining the mechanisms of

AHL by re-analyzing the microarray data of Kwon et al. (Kwon et al. 2015) through addition of network-related bioinformatics algorithms. In addition, small molecule drugs were also predicted in order to find potential treatments for AHL.

Materials and methods

\section{Microarray data}

The microarray data under accession number GSE70659 were collected from the Gene

(Supplemental Information 1), which contained cochlear tissues from wild-type (WT, $\mathrm{n}=3$ ) and

Cmah-null ( $\mathrm{n}=3$ ) C57BL/6J mice with old age (12 months).

\section{Data normalization and DEGs identification}


transformation and quantile normalization) using the lumiR package in R (www.r-project.org).

127 The DEGs between WT and Cmah-null mice were identified using the Linear Models for

128 Microarray data (LIMMA) method (Smyth 2005) in the Bioconductor R package

129 (http://www.bioconductor.org/packages/release/bioc/html/limma.html). After the t-test, and the

130 p-value was multiple corrected with the Benjamini-Hochberg $(\mathrm{BH})$ procedure (Benjamini \&

131 Hochberg 1995). Genes were considered to be significantly differentially at $\mathrm{p}<0.05$ and

$132 \mid \log \mathrm{FC}($ fold change $) \mid>0.5$ due to the poor BH-adjusted p-value.

133

134

135

136

137

138

139

140

141

142

143

144

145

146

\section{Protein-protein interaction (PPI) network construction}

The PPI pairs were downloaded from acknowledged STRING 10.0 (Search Tool for the

Retrieval of Interacting Genes; http://string db.org/) database (Szklarczyk et al. 2015) and then

the DEGs were imported into the PPI data to obtain the whole PPI network. The PPIs with

combined scores $>0.4$ were selected to construct the PPI network which was visualized using the

Cytoscape software (version 2.8; www.cytoscape.org/) (Kohl et al. 2011). The crucial nodes within PPI network were analyzed based on three topological properties using the CytoNCA plugin in Cytoscape software (http://apps.cytoscape.org/apps/cytonca) (Tang et al. 2015), including degree [the number of interactions per node (protein)], betweenness (the number of shortest paths that pass through each node) and closeness centrality (the average length of the shortest paths to access all other proteins in the network). Functionally related and densely interconnected clusters were extracted from the large PPI network using the Molecular Complex

Detection (MCODE) plugin of Cytoscape software according to the following parameters: degree cutoff $=5$; node score cutoff $=0.4 ; \mathrm{k}$-core $=5 ;$ and maximum depth $=100$ 
147 (ftp://ftp.mshri.on.ca/pub/BIND/Tools/MCODE) (Bader \& Hogue 2003). Modules were 148 considered significant with MCODE score $\geq 4$ and nodes $\geq 6$.

149 Furthermore, whether the DEGs were transcription factors (TFs) and the TF-target gene 150 interactions were predicted by the TRANSFAC database (http://www.gene151 regulation.com/pub/databases.html) (Matys et al. 2006), and then were integrated into the PPI 152 network to establish a regulatory network.

153 Function enrichment analysis

154 Kyoto encyclopedia of genes and genomes (KEGG) pathway enrichment analyses were 155 performed to investigate the underlying functions of all DEGs and DEGs in the network using 156 The Database for Annotation, Visualization and Integrated Discovery (DAVID) online tool 157 (version 6.8; http://david.abcc.ncifcrf.gov) (Huang et al. 2009). P-value < 0.05 was set as the cut158 off value.

159

160 161 162 163 164 165 166 167

\section{miRNA-target gene regulatory network construction}

The miRNAs that can regulate the DEGs in the PPI network were predicted using the miRWalk database (version 2.0; http: //www.zmf.umm.uni-heidelberg.de/apps/zmf/mirwalk2) with default significant parameters. Only the interaction relationships can be predicted by nine common algorithms, including miRWalk, Microt4, miRanda, miRDB, miRMap, miRNAMap, RNA22, RNAhybrid and Targetscan, were included to construct the miRNA-target gene regulatory network using the Cytoscape software (Kohl et al. 2011).

\section{Screening of small-molecule drugs for treatment of $A H L$}

The name of DEGs identified in PPI network were converted to HG-U133A probe set IDs 
168

169

170

171

172

173

174

175

176

177

and uploaded to the Connectivity Map (CMAP, http://www.broadinstitute.org/cmap/) database which is a collection of genome-wide transcriptional expression data from human cancer cell lines treated with bioactive small molecules. If the enrichment score was close to -1 , the corresponding small molecules were the potential drugs to reverse the expression of the query signature. Significant small-molecule drugs were selected according to the threshold value of $p<$ 0.05 and $\mid$ mean $\mid>0.4$.

\section{Results}

\section{Identification of DEGs}

After data normalization, 799 genes were identified as DEGs between WT and Cmah-null mice based on the threshold of $\mathrm{p}<0.05$ and $|\operatorname{logFC}|>0.5$, including 449 upregulated (such as Fos, FBJ osteosarcoma oncogene) and 350 downregulated genes (such as Ucp1, uncoupling protein 1 (mitochondrial, proton carrier); Acadm, acyl-Coenzyme A dehydrogenase, medium chain). All the DEGs are listed in Supplemental Information 2. As shown in Figure 1, heat map illustrated that the expression patterns of genes were obviously altered in Cmah-knockout mice compared with control.

\section{Function enrichment analysis of DEGs}

The above differential genes were subjected to the DAVID for function enrichment analysis. As a result, 16 KEGG pathways were enriched for upregulated DEGs, including ECM-receptor interaction (LAMA1, laminin, alpha 1; ITGA5, integrin subunit alpha 5), Cell adhesion molecules (ICAM1, intercellular adhesion molecule 1; ITGA5), Focal adhesion (LAMA1; 
188 ITGA5), Cytokine-cytokine receptor interaction (TNFSF13B, TNF superfamily member 13b) 189 and TNF signaling pathway (FOS; ICAM1); while 20 KEGG pathways were for downregulated DEGs, including Oxidative phosphorylation (UQCRC2, ubiquinol cytochrome c reductase core protein 2), metabolism related and PPAR signaling pathway (ACADM; PPARG, Peroxisome proliferator-activated receptor gamma; UCP1; SCD1, stearoyl-Coenzyme A desaturase 1) (Table 193 $1)$.

\section{PPI network construction}

After mapping the DEGs into the protein interactions downloaded from the STRING database, a PPI network was constructed (Figure 2) which included 587 nodes (303 upregulated and 284 downregulated; 52 TFs) and 2944 edges (interaction relationships) (Supplemental Information 3). UQCRC2, SOD2 (superoxide dismutase 2), FOS, ICAM1 and PPARG were suggested to be hub genes by calculating the degree, betweenness, and closeness centrality of nodes in the PPI network. Among them, FOS and PPARG were TFs (Table 2) and they both interacted with ICAM1. Furthermore, PPARG also could interact with FOS and SOD2.

After cluster analysis according to the given parameters, two significant modules were obtained (Table 3). Function enrichment analysis showed that the genes in module 1 (Figure 3) were closely related to Oxidative phosphorylation (UQCRC2), while the genes in module 2

(Figure 4) were significantly enriched in metabolism related and PPAR signaling pathway (ACADM) (Table 4).

miRNA-target gene regulatory network analysis

Using the miRWalk2.0 database, 82 DEGs were predicted to be regulated by 193 miRNAs 
209 in 9 databases (Supplemental Information 4). Then, 166 interaction relationships between 43

210 upregulated DEGs and 117 miRNAs as well as 182 interaction relationships between 39

211 downregulated DEGs and 76 miRNAs were used for constructing the upregulated (Figure 5) and

212 downregulated (Figure 6) miRNA-mRNA regulatory network, respectively. As shown in figure 5,

213 FOS can be regulated by mmu-miR-221-3p or mmu-miR-222-3p, while PPARG can be

214 regulated by mmu-miR-130b-3p, mmu-miR-27a-3p, mmu-miR-27b-3p and mmu-miR-721 in

215 figure 6.

216 Small-molecule drugs

217 The DEGs in PPI network were uploaded into CMAP database to obtain the small-molecule

218 drugs. As a result, 69 small-molecule chemicals with negative mean and enrichment scores were

219 predicted, such as adiphenine, DL-PPMP, decitabine and topiramate. This finding indicated their

220 potential ability to inhibit the development of AHL (Table 5).

\section{Discussion}

In the present study, Cmah-null mice were used as an animal model to investigate the underlying mechanisms of AHL. In line with the study of Kwon et al. (Kwon et al. 2015), oxidative phosphorylation pathway was enriched in the DEGs between Cmah-null mice and WT, and SOD2, SIRT3 were crucial genes in PPI network (Table 3), further demonstrating the oxidative stress pathogenesis of AHL (Fujimoto \& Yamasoba 2014). In addition, our current 227 study also found ECM-receptor interaction (LAMA1), adhesion (ICAM1), inflammation (FOS, 228 ICAM1, TNFSF13B) and PPAR signaling pathways (PPARG). Among them, PPAR signaling 
229 pathway may be especially important because the following causes: 1) this pathway was 230 enriched for the genes in PPI and significant modules; 2) PPARG was a TF; and 3) PPARG 231 could interact with SOD2, FOS and ICAM1. Furthermore, we predicted PPARG can be 232 regulated by mmu-miR-130b-3p, mmu-miR-27a-3p, mmu-miR-27b-3p and mmu-miR-721. Also, 233 adiphenine, DL-PPMP, decitabine and topiramate were speculated to be potential small-molecule 234 drugs to reverse the expression of PPARG in AHL. Accordingly, we hypothesize downregulated PPARG may be involved in AHL by influencing adhesion, inflammation and oxidative stress, while downregulation of the above miRNAs and the use of the above small-molecule drugs may be potential treatment strategies for AHL by upregulating PPARG.

The extracellular matrix (ECM), the non-cellular component throughout all tissues and organs, and adherens junctions between cells and ECM are essential for maintenance of the structural and functional integrity of organs. ECM (i.e. laminin, integrin, fibronectin or collagen) and adherens (i.e. cadherin, syndecan-1, tenascin-C, Connexin or Icam) molecules are suggested to play a vital role for the growth and proliferation of cochlear sensorineural epithelial cells and sensory cell synaptogenesis (Evans et al. 2007; Toyama et al. 2005; Wang et al. 2015). The expression changes in the above molecules may cause hearing loss. For example, Suzuki et al. found type IX collagen knockout mice exhibited abnormal integrity of collagen fibers in the tectorial membrane and showed progressive hearing loss by auditory brainstem response assessment (Suzuki et al. 2005). Gottesberge et al. demonstrated deletion of the discoidin domain receptor 1 (DDR1) in mouse, a tyrosine kinase receptor activated by native collagen, induced deterioration of the supporting cells and consequently interfere with mechanical properties of the 
250

251

252

254

256

257

258

259

260

261

262

263

264

265

266

267

268

269

270

organ of Corti, leading to a severe decrease in auditory function (Gottesberge et al. 2008). Cai et al. proved that exposure to an intense noise for $2 \mathrm{~h}$ caused site-specific changes in expression levels of genes from adhesion families in the apical (upregulated: Sell, Thbs1, Itgae, Icam1, and Itga5) and the basal (upregulated: Itga3, Itgb2, Selp, Sele, Cdh1, and Cdh2) sections of the sensory epithelium in the cochlea. Selp and Itga5 in the basal section were positively, but Sell in the apical section was negatively correlated with greater hearing loss (Cai et al. 2012). In line with the above findings, our present study also identified several ECM and adherens genes to be differentially expressed, with the upregulation of LAMA1, ICAM1 and ITGA5, suggesting these genes may be underlying targets for treatment of AHL. Our hypothesis had been preliminarily demonstrated in the study of Ramunni et al. who found inhibition of adhesion molecules (sEselectin, sVCAM-1 and sICAM-1) by a single session of LDL/fibrinogen apheresis led to a complete hearing recovery (Ramunni et al. 2006).

In addition, the upregulation of adherens genes may favor the interaction between leukocytes and inner ear endothelial cells, promoting the inflammation and hearing loss (Kanzaki et al. 2014; Ramunni et al. 2006), indicating inflammation related pathways may also be a target for AHL. In accordance with our expected, TNF signaling pathway and Cytokine-cytokine receptor interaction pathways were also significantly enriched for upregulated genes (TNFSF13B; FOS; ICAM1) in this study. It had been reported that TNF- $\alpha$ and its receptors (TNFR1, TNFR2) were higher expressed in the cochlea of vibration- or noise-induced hearing loss (Fuentessantamaría et al. 2017; Zou et al. 2005). Use of TNF- $\alpha$ inhibitor preserved the hearing threshold by improvement of cochlear blood flow (Arpornchayanon et al. 2013). The expression 
271 of FOS was found to be dynamically changed after deafness, with lower level in the auditory

272 cortex 15 days (compensation mechanism), but increased from 2 weeks and stabilized three

273 months after permanent auditory deprivation in adult rats (Pernia et al. 2017). As a TF, FOS may

274 participate in inflammation by regulating its target genes, such as ICAM-1, CSF1 and CCL5

275 which were all important inflammatory proteins for hearing loss (Trune et al. 2015).

PPARs are ligand-activated TFs belonging to the nuclear receptor superfamily. Extensive

277 studies have shown that PPAR participates in various biological functions such as cell

proliferation, apoptosis and differentiation by regulating its target genes (Chung et al. 2008). For

example, there is evidence to reveal that PPAR expression is inversely correlated with inflammatory cytokines IL-1 $\beta$ and TNF- $\alpha$ in aging rats (Gelinas \& Mclaurin 2005). The PPAR $\gamma$ agonist ameliorates aging-related renal and cerebral artery injuries by inhibiting the inflammatory genes, reducing ECM production, and attenuating oxidative stress (Sung et al. 2006; Wang et al. 2014; Yang et al. 2009). In this study, we also found PPAR $\gamma$ was downregulated in the cochlear tissues of Cmah-null mice and our PPI network showed PPARG could interact with SOD2, FOS and ICAM1, implying PPARG mediated pathways may be also a considerably important mechanism for AHL and activation of PPARG may be an underlying therapeutic method for patients with AHL, which has not been reported previously. the expression of target genes via binding to the 3'-untranslated region (UTR) and then participate in the cellular processes. There has been evidence to indicate miRNAs participate in the pathogenesis of AHL, including miR-34a (Huang et al. 2017; Pang et al. 2017) and miR-29b 
292 (Xue et al. 2016). These two miRNAs were involved in AHL by regulating ROS homeostasis293 related gene SIRT1 and then influencing cochlear hair cell apoptosis. However, as a crucial gene 294 identified to be associated with ROS in AHL of our study, there was no study to investigate the 295 miRNAs that regulate PPARG in AHL. Thus, we also predicted the potential miRNAs that 296 regulate PPARG by using the miRwalk database. As a result, miR-130b-3p, miR-27a-3p, miR$29727 b-3 p$ and miR-721 were screened. miR-27b-3p has been shown to target PPARG to inhibit cell proliferation, but increase the inflammatory response to promote cell apoptosis (Lee et al. 2012). Nevertheless, there were no studies on the relationship between PPARG and others miRNAs in cell apoptosis, which may be our future research direction.

Furthermore, we also identified the potential drugs for inhibiting PPARG, consisting of the most negatively correlated adiphenine, DL-PPMP, decitabine and topiramate. Several studies have demonstrated topiramate could attenuate oxidative damage, inflammation and neuronal cell death (Motaghinejad M 2016; Tian et al. 2015), indicating topiramate may also be an underlying drug for PPARG-related AHL.

However, there were some limitations in this study. First, the sample size in the microarray 307 data GSE70659 was small. Another microarray or sequencing experiments should be performed

to further screen crucial mechanisms for AHL. Second, we only preliminarily identified the AHL-related genes, miRNAs and drugs. Additional in vivo and in vitro experiments (PCR, Western blotting, knockout and overexpression design) are necessary to confirm their expression and their functions.

\section{Conclusion}


313 Our present study preliminarily reveals Cmah deficiency may lead to AHL by 314 downregulating PPARG, which may then induce the higher expressions of ECM and adhesion 315 (ICAM1) and pro-inflammatory (FOS, TNFSF13B), but lower expression of anti-oxidative genes 316 (SOD2). Downregulation of miR-130b-3p, miR-27, miR-721 and the use of topiramate may be 317 potential treatment strategies for ALH by upregulating PPARG.

318

319

320

321

322

323

\section{Availability of data and materials}

Raw data is available in the Supplemental Materials (Supplemental Information 1).

\section{References}

Arpornchayanon W, Canis M, Ihler F, Settevendemie C, and Strieth S. 2013. TNF- $\alpha$ inhibition using etanercept prevents noise-induced hearing loss by improvement of cochlear blood flow in vivo. Int $J$ Audiol 52:545-552.

Bader GD, and Hogue CW. 2003. An automated method for finding molecular complexes in large protein interaction networks. BMC Bioinformatics 4:2.

Benjamini Y, and Hochberg Y. 1995. Controlling the false discovery rate: A practical and powerful approach to multiple testing. JR Stat Soc B 57: 289-300.

Cai Q, Patel M, Coling D, and Hu BH. 2012. Transcriptional changes in adhesion-related genes are site-specific during noise-induced cochlear pathogenesis. Neurobiol Dis 45:723-732.

Chung J, Seo A, Chung S, Kim M, Leeuwenburgh C, Yu B, and Chung H. 2008. Molecular mechanism of PPAR in the regulation of age-related inflammation. Ageing Res Rev 7:126-136. 
333 Ciorba A, Bianchini C, Pelucchi S, and Pastore A. 2012. The impact of hearing loss on the quality of life of elderly adults. Clin Interv Aging 7:159.

335

336

337

338

339

340

341

342

343

344

345

346

347

348

349

350

351

352

353 antioxidant enzymes related to hydrogen peroxide metabolism in rat inner ear. Neurosci Lett 464:22-25.

Ding D, Jiang H, Chen GD, Longo-Guess C, Muthaiah VP, Tian C, Sheppard A, Salvi R, and Johnson KR. 2016. N-acetyl-cysteine prevents age-related hearing loss and the progressive loss of inner hair cells in $\gamma$-glutamyl transferase 1 deficient mice. Aging 8:730-750.

Dong Y, Guo CR, Ding Y, Zhang Y, Song HY, Peng YT, Zhang T, and Shi JR. 2016. Effects of Erlong Zuoci decoction on the age-related hearing loss in C57BL/6J mice. $J$ Ethnopharmacol 181:59-65.

Du Z, Yang Q, Liu L, Li S, Zhao J, Hu J, Liu C, Qian D, and Gao C. 2015. NADPH oxidase 2dependent oxidative stress, mitochondrial damage and apoptosis in the ventral cochlear nucleus of D-galactose-induced aging rats. Neuroscience 286:281-292.

Evans AR, Euteneuer S, Chavez E, Mullen LM, Hui EE, Bhatia SN, and Ryan AF. 2007. Laminin and fibronectin modulate inner ear spiral ganglion neurite outgrowth in an in vitro alternate choice assay. Dev Neurobiol 67:1721-1730.

Fuentessantamaría V, Alvarado JC, Melgarrojas P, Gabaldónull MC, Miller JM, and Juiz JM. 2017. The Role of Glia in the Peripheral and Central Auditory System Following Noise Overexposure: Contribution of TNF- $\alpha$ and IL-1 $\beta$ to the Pathogenesis of Hearing Loss. 
355

356

357

358

359

360

361

362

363

364

365

366

367

368

369

370

371

372

373

374

Fujimoto C, and Yamasoba T. 2014. Oxidative stresses and mitochondrial dysfunction in agerelated hearing loss. Oxid Med Cell Longev 2014:582849.

Gelinas DS, and Mclaurin JA. 2005. PPAR- $\alpha$ Expression Inversely Correlates with Inflammatory Cytokines IL-1 $\beta$ and TNF- $\alpha$ in Aging Rats. Neurochem Res 30:1369-1375.

Go S, Yoshikawa M, Inokuchi J. 2011. Glycoconjugates in the mammalian auditory system. J Neurochem 116:756-763.

Gottesberge AMMZ, Gross O, Beckerlendzian U, Massing T, and Vogel WF. 2008. Inner ear defects and hearing loss in mice lacking the collagen receptor DDR1. Lab Invest 88:2737.

Hedlund M, Tangvoranuntakul P, Takematsu H, Long JM, Housley GD, Kozutsumi Y, Suzuki A, Wynshawboris A, Ryan AF, and Gallo RL. 2007. N-Glycolylneuraminic Acid Deficiency in Mice: Implications for Human Biology and Evolution. Mol Cell Biol 27:4340-4346.

Hjalte F, Brännström J, and Gerdtham UG. 2012 Societal costs of hearing disorders: a systematic and critical review of literature. Int J Audiol 51: 655-662.

Huang DW, Sherman BT, and Lempicki RA. 2009. Systematic and integrative analysis of large gene lists using DAVID bioinformatics resources. Nat Protoc 4:44.

Huang Q, Xiong H, Yang H, Ou Y, Zhang Z, Chen S, Ye Y, and Zheng Y. 2016. Differential Expression of Bcl-2 in the Cochlea and Auditory Cortex of a Mouse Model of AgeRelated Hearing Loss. Audiol Neurootol 21:326-332.

Huang Q, Zheng Y, Ou Y, Xiong H, Yang H, Zhang Z, Chen S, and Ye Y. 2017. miR-34a/Bcl-2 
375

376

377

378

379

380

381

382

383

384

385

386

387

388

389

390

391

392

393

394

395

signaling pathway contributes to age-related hearing loss by modulating hair cell apoptosis. Neurosci Lett 661:51-56.

Hwang JH, Chen JC, Hsu CJ, Yang WS, and Liu TC. 2012. Plasma reactive oxygen species levels are correlated with severity of age-related hearing impairment in humans. Neurobiol Aging 33:1920-1926.

Inokuchi JI, Go S, Yoshikawa M, Strauss K. 2017. Biochim Biophys Acta Gen Subj 1861:24852493.

Kang JW, Choi HS, Kim K, and Choi JY. 2014. Dietary vitamin intake correlates with hearing thresholds in the older population: the Korean National Health and Nutrition Examination Survey. Am J Clin Nutr 99:1407-1413.

Kanzaki S, Sakagami M, Hosoi H, Murakami S, and Ogawa K. 2014. High Fibrinogen in Peripheral Blood Correlates with Poorer Hearing Recovery in Idiopathic Sudden Sensorineural Hearing Loss. Plos One 9:e104680.

Kohl M, Wiese S, and Warscheid B. 2011. Cytoscape: software for visualization and analysis of biological networks. Methods Mol Biol 696: 291-303.

Kwon DN, Park WJ, Choi YJ, Gurunathan S, Kim JH. 2015. Oxidative stress and ROS metabolism via down-regulation of sirtuin 3 expression in Cmah-null mice affect hearing loss. Aging 7:579-594.

Lasisi AO, Fehintola FA. 2011. Correlation between plasma levels of radical scavengers and hearing threshold among elderly subjects with age-related hearing loss. Acta Otolaryngol 
396

397

398

399

400

401

402

403

404

405

406

407

408

409

410

411

412

413

414

415

416

Lasisi TJ, and Lasisi AO. 2015. Evaluation of serum antioxidants in age-related hearing loss. Aging Clin Exp Res 27:265-269.

Lee JJ, Drakaki A, Iliopoulos D, and Struhl K. 2012 MiR-27b targets PPAR $\gamma$ to inhibit growth, tumor progression and the inflammatory response in neuroblastoma cells. Oncogene 31: 3818-3825.

Markaryan A, Nelson EG, and Raul Hinojosa MD. 2009. Quantification of the mitochondrial DNA common deletion in presbycusis. Laryngoscope 119:1184.

Matys V, Kelmargoulis OV, Fricke E, Liebich I, Land S, Barredirrie A, Reuter I, Chekmenev D, Krull M, and Hornischer K. 2006. TRANSFAC® and its module TRANSCompel ${ }^{\circledR}$ : transcriptional gene regulation in eukaryotes. Nucleic Acids Res 34:D108-D110.

Menardo J, Tang Y, Ladrech S, Lenoir M, Casas F, Michel C, Bourien J, Ruel J, Rebillard G, and Maurice T. 2012. Oxidative stress, inflammation, and autophagic stress as the key mechanisms of premature age-related hearing loss in SAMP8 mouse Cochlea. Antioxid Redox Signal 16:263-274.

Motaghinejad M MM, Shabab B. 2016. Neuroprotective effects of various doses of topiramate against methylphenidate induced oxidative stress and inflammation in rat isolated hippocampus. Clin Exp Pharmacol Physiol 43:360-371.

Niklaus RT, Dirk H, and Rudolf P. 2011. Prevalence of age-related hearing loss in Europe: a review. Eur Arch Oto-Rhino-L 268:1101-1107.

Pang J, Xiong H, Lin P, Lai L, Yang H, Liu Y, Huang Q, Chen S, Ye Y, and Sun Y. 2017. Activation of miR-34a impairs autophagic flux and promotes cochlear cell death via 
repressing ATG9A: implications for age-related hearing loss. Cell Death Dis 8:e3079.

418

419

420

421

422

423

424

425

426

427

428

429

430

431

432

433

434

435

436

437

Peelle JE, and Wingfield A. 2016. The neural consequences of age-related hearing loss. Trends Neurosci 39:486-497.

Pernia M, Estevez S, Poveda C, Plaza I, Carro J, Juiz M, and Merchan MA. 2017. c-Fos and Arc/Arg3.1 expression in auditory and visual cortices after hearing loss: Evidence of sensory crossmodal reorganization in adult rats. J Comp Neuro 525:2677-2689.

Quaranta N, Coppola F, Casulli M, Barulli MR, Panza F, Tortelli R, Solfrizzi V, Sabbà C, and Logroscino G. 2015. Epidemiology of age related hearing loss: A review. Hearing Balance Commun 13: 77-81.

Ramunni A, Quaranta N, Saliani MT, Fallacara RA, Ria R, and Ranieri G. 2006. Does a reduction of adhesion molecules by LDL-apheresis have a role in the treatment of sudden hearing loss? Ther Apher Dial 10:282-286.

Riva C, Donadieu E, Magnan J, and Lavieille JP. 2007. Age-related hearing loss in CD/1 mice is associated to ROS formation and HIF target proteins up-regulation in the cochlea. Exp Gerontol 42:327-336.

Smyth GK. 2005. Limma: linear models for microarray data. Bioinformatics and computational biology solutions using R and Bioconductor: Springer, 397-420.

Someya S, Tanokura M, Weindruch R, Prolla TA, and Yamasoba T. 2010a. Effects of caloric restriction on age-related hearing loss in rodents and rhesus monkeys. Curr Aging Sci $3: 20-25$.

Someya S, Yu W, Hallows WC, Xu J, Vann JM, Leeuwenburgh C, Tanokura M, Denu JM, and 
438

439

440

441

442

443

444

445

446

447

448

449

450

451

452

453

454

455

456

457

458

Prolla TA. 2010b. Sirt3 Mediates Reduction of Oxidative Damage and Prevention of Age-related Hearing Loss under Caloric Restriction. Cell 143:802-812.

Sung B, Park S, Yu BP, and Chung HY. 2006. Amelioration of age-related inflammation and oxidative stress by PPARgamma activator: suppression of NF-kappaB by 2,4thiazolidinedione. Exp Gerontol 41:590-599.

Suzuki N, Asamura K, Kikuchi Y, Takumi Y, Abe S, Imamura Y, Hayashi T, Aszodi A, Fässler R, and Usami SI. 2005. Type IX collagen knock-out mouse shows progressive hearing loss. Neurosci Res 51:293-298.

Szklarczyk D, Franceschini A, Wyder S, Forslund K, Heller D, Huerta-Cepas J, Simonovic M, Roth A, Santos A, and Tsafou KP. 2015. STRING v10: protein-protein interaction networks, integrated over the tree of life. Nucleic Acids Res 43:D447.

Tang Y, Li M, Wang J, Pan Y, and Wu FX. 2015. CytoNCA: a cytoscape plugin for centrality analysis and evaluation of protein interaction networks. Biosystems 127:67-72.

Tian Y, Guo SX, Li JR, Du HG, Wang CH, Zhang JM, and Wu Q. 2015. Topiramate attenuates early brain injury following subarachnoid haemorrhage in rats via duplex protection against inflammation and neuronal cell death. Brain Res 1622:174-185.

Toyama K, Ozeki M, Hamajima Y, and Lin J. 2005. Expression of the integrin genes in the developing cochlea of rats. Hear Res 201:21-26.

Trune DR, Kempton B, Hausman FA, Larrain BE, and Macarthur CJ. 2015. Correlative mRNA and Protein Expression of Middle and Inner Ear Inflammatory Cytokines during Mouse Acute Otitis Media. Hear Res 326:49-58. 
459 Wang B, Hu B, and Yang S. 2015. Cell junction proteins within the cochlea: A review of recent research. J Otol 10:131-135.

461 Wang P, Li B, Cai G, Huang M, Jiang L, Pu J, Li L, Wu Q, Zuo L, and Wang Q. 2014. Activation of PPAR- $\gamma$ by pioglitazone attenuates oxidative stress in aging rat cerebral arteries through upregulating UCP2. J Cardiovasc Pharmacol 64:497-506.

464

Watanabe K, Shibuya S, Ozawa Y, Nojiri H, Izuo N, Yokote K, and Shimizu T. 2014. Superoxide dismutase 1 loss disturbs intracellular redox signaling, resulting in global age-related pathological changes. Biomed Res Int 2014:140165.

Xue T, Wei L, Zha DJ, Qiu JH, Chen FQ, Qiao L, and Qiu Y. 2016. miR-29b overexpression induces cochlear hair cell apoptosis through the regulation of SIRT1/PGC-1 $\alpha$ signaling: Implications for age-related hearing loss. Int J Mol Med 38:1387-1394.

Yamasoba T, Someya S, Yamada C, Weindruch R, Prolla TA, and Tanokura M. 2007. Role of mitochondrial dysfunction and mitochondrial DNA mutations in age-related hearing loss. Hear Res 226:185.

Yang HC, Deleuze S, Zuo Y, Potthoff SA, Ma LJ, and Fogo AB. 2009. The PPAR $\gamma$ Agonist Pioglitazone Ameliorates Aging-Related Progressive Renal Injury. J Am Soc Nephrol $20: 2380-2388$.

Zeng L, Yang Y, Hu Y, Sun Y, Du Z, Xie Z, Zhou T, and Kong W. 2014. Age-Related Decrease in the Mitochondrial Sirtuin Deacetylase Sirt3 Expression Associated with ROS Accumulation in the Auditory Cortex of the Mimetic Aging Rat Model. Plos One 9:e88019. 
480 Zou J, Pyykkö I, Sutinen P, and Toppila E. 2005. Vibration induced hearing loss in guinea pig 481 cochlea: expression of TNF- $\alpha$ and VEGF. Hear Res 202:13-20.

482

483

484

485

486

487

488

489

490

491

492

493

494

495

496

497

498

499

500

Figure legends:

Peer] reviewing PDF | (2019:01:34403:1:1:NEW 18 Mar 2019) 
501 Figure 1. Heat map of differentially expressed genes in cochlear tissues from wild-type (WT) 502 and Cmah-null C57BL/6J mice with old age. WT1-3 or Cmah-null 1-3 indicated three repeats in 503 each group. The heat map indicates that the samples of Cmah knockout and control mice could 504 be completely separated by the differentially expressed genes. Green, low expression; red, high 505 expression.

Figure 2. The protein-protein interaction network. The red and green nodes represent the upregulated and downregulated genes, respectively. Triangle, transcription factors; circular, 508 mRNA.

Figure 3. The significant module 1 extracted from protein-protein interaction network. The green nodes represent downregulated genes.

Figure 4. The most significant module 2 extracted from protein-protein interaction network. The red and green nodes represent the upregulated and downregulated genes, respectively.

Figure 5 The miRNA-mRNA regulatory network for the upregulated genes. Blue: miRNAs; purple: upregulated genes; hazel-green: upregulated transcription factors.

Figure 6 The miRNA-mRNA regulatory network for the downregulated genes. Blue: miRNAs; purple: downregulated genes; hazel-green: downregulated transcription factors.

Supplementary Materials:

Supplemental Information 1: Raw data used in this manuscript. 
522 Supplemental Information 2: All differentially expressed genes.

523 Supplemental Information 3: Protein-protein interaction data.

524 Supplemental Information 4: miRNA-mRNA interaction prediction results

525

526 
Figure 1

Heat map of differentially expressed genes between Cmah-null and wild-type mice 


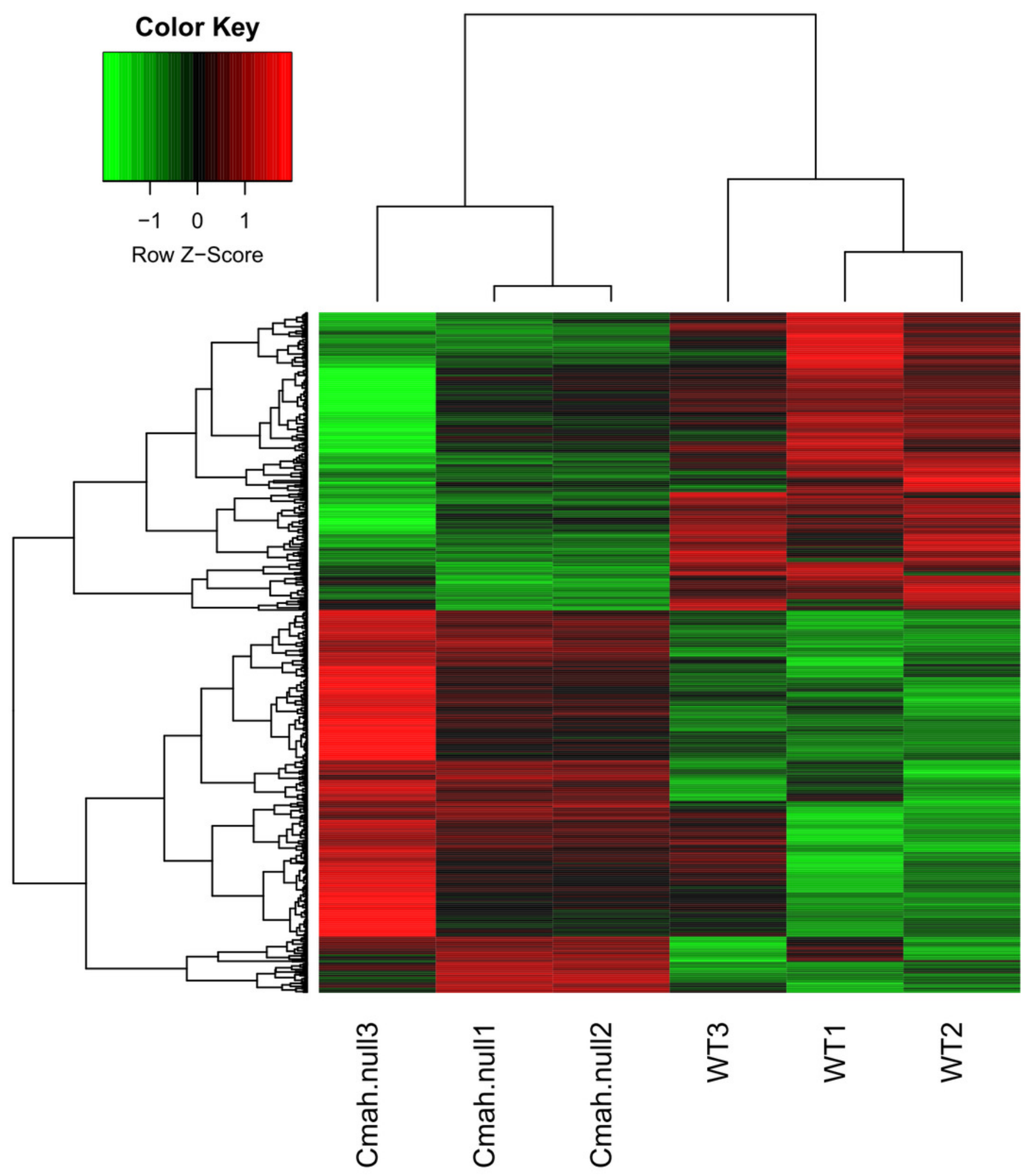


Figure 2

The protein-protein interaction network.

The red and green nodes represent the upregulated and downregulated genes, respectively. Triangle, transcription factors; circular, mRNA.

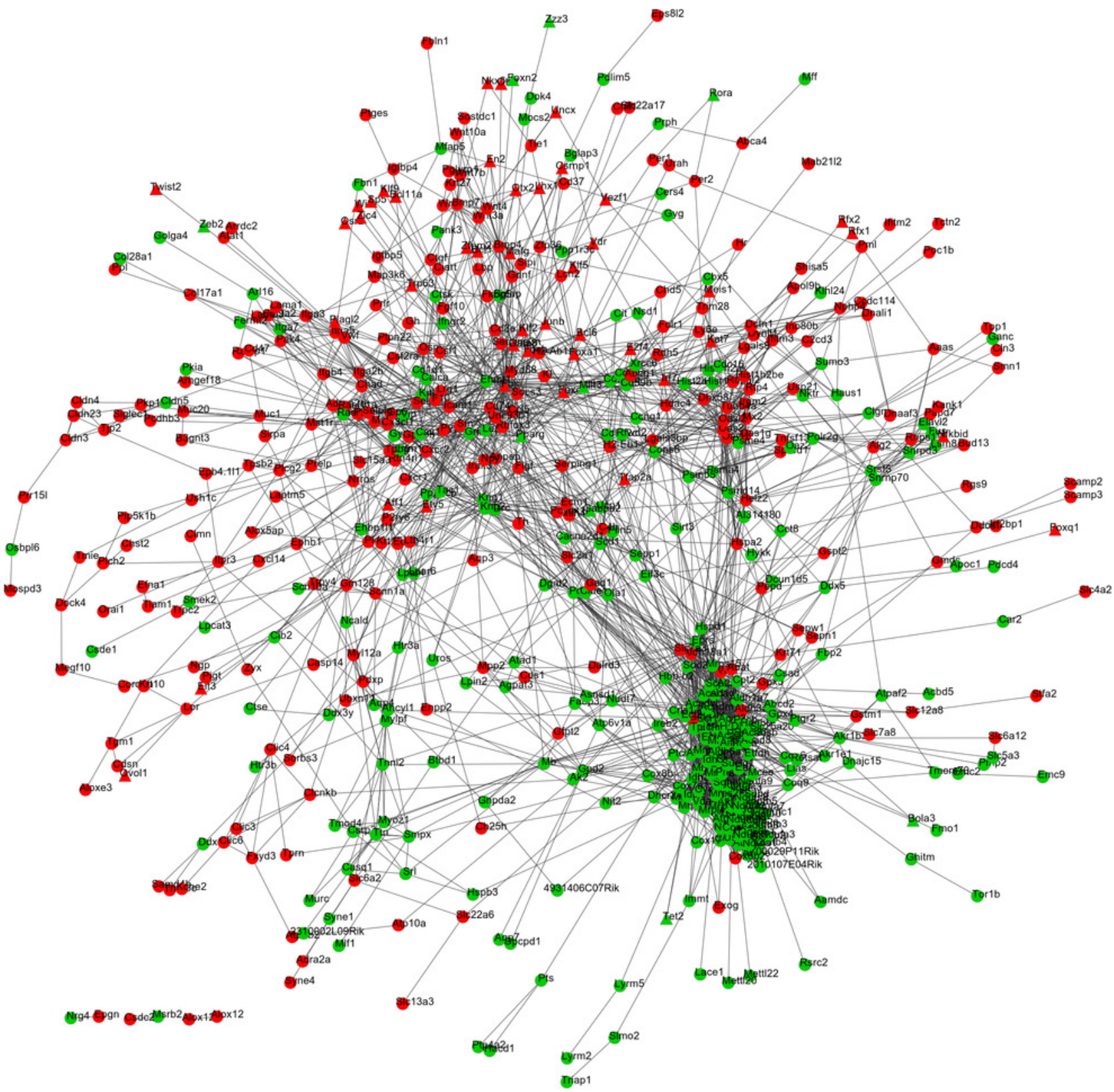


Figure 3

The significant module 1 extracted from protein-protein interaction network.

The green nodes represent downregulated genes.

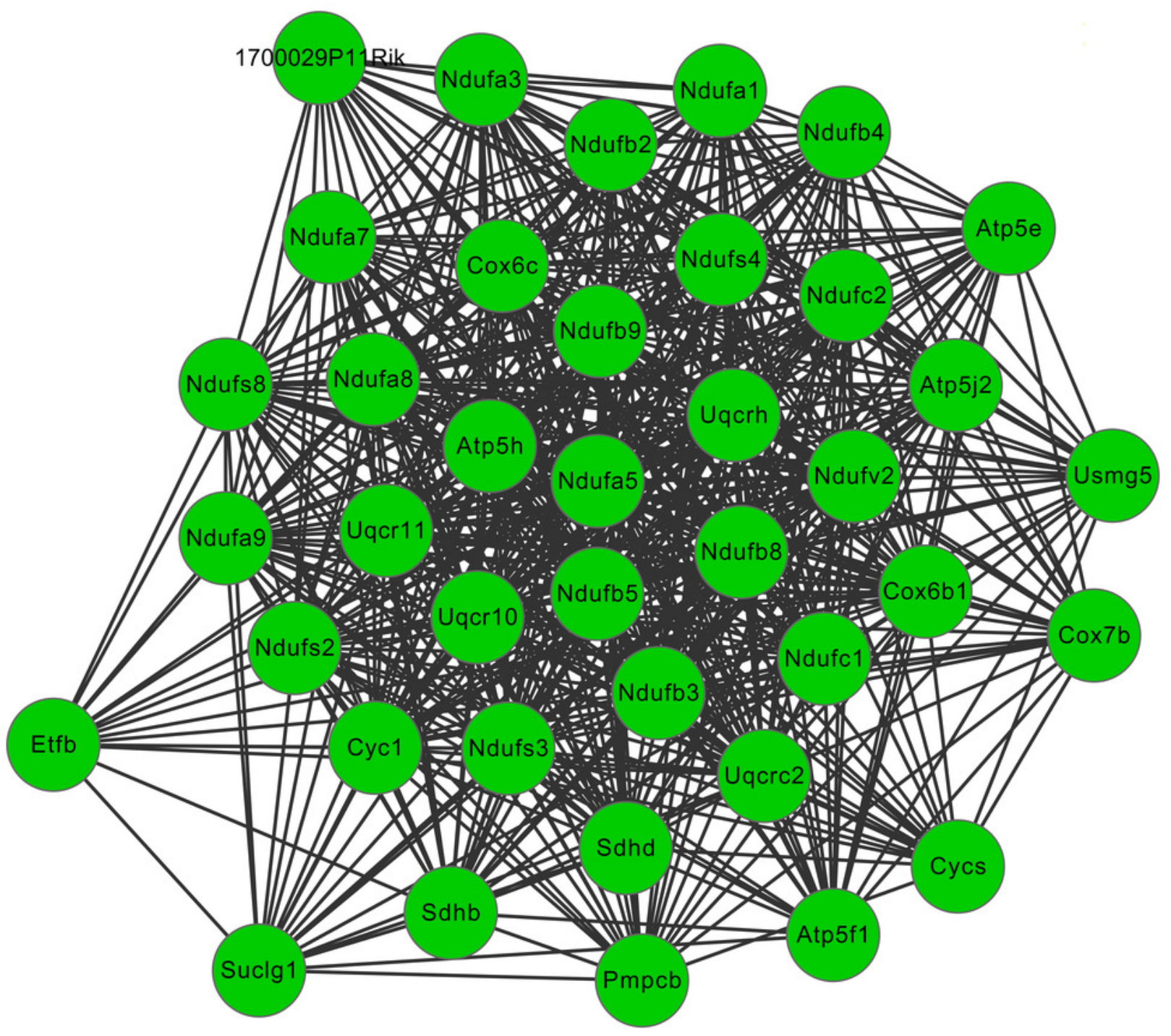


Figure 4

The most significant module 2 extracted from protein-protein interaction network

The red and green nodes represent the upregulated and downregulated genes, respectively.

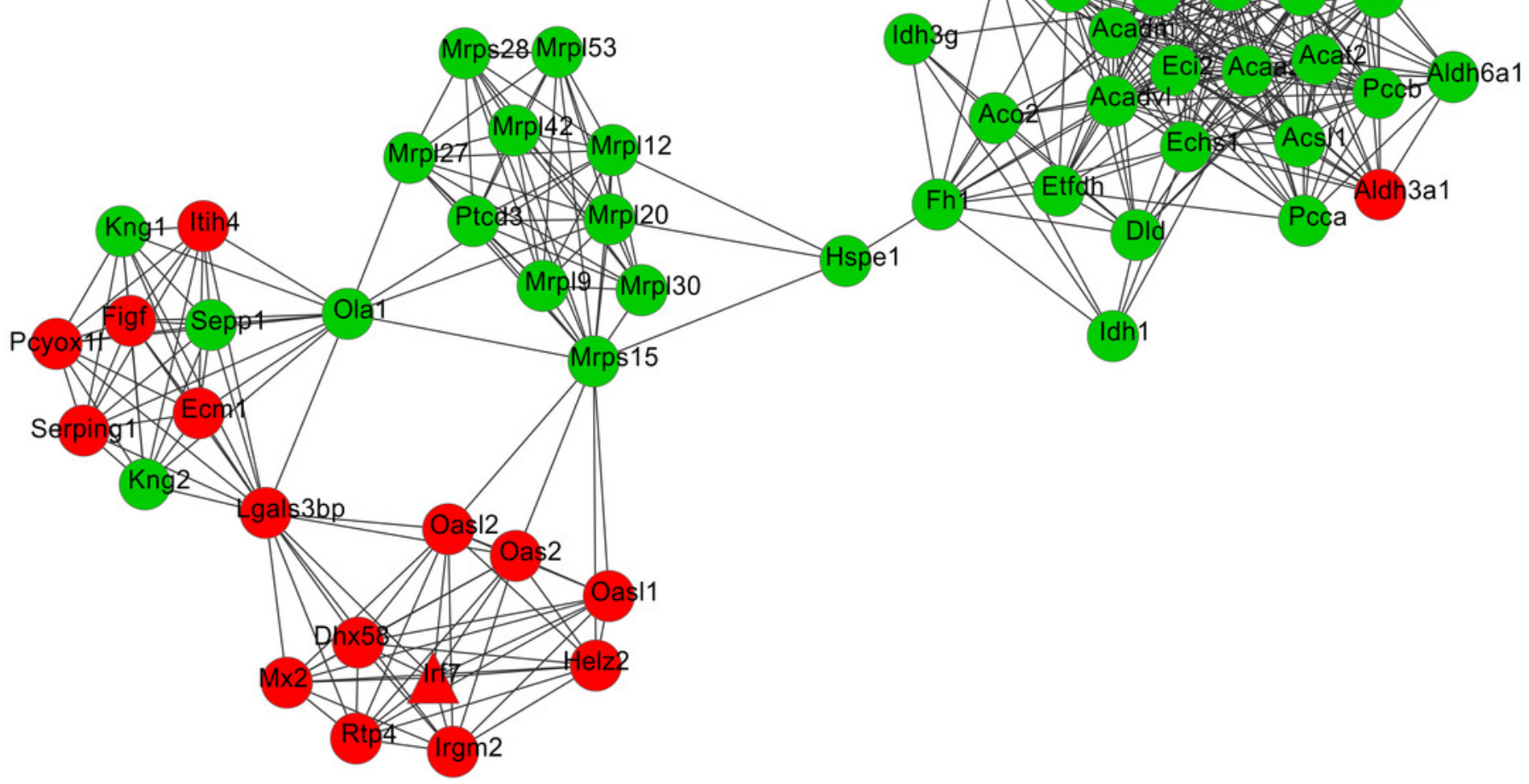


Figure 5

The miRNA-mRNA regulatory network for the upregulated genes.

Blue: miRNAs; purple: upregulated genes; hazel-green: upregulated transcription factors.

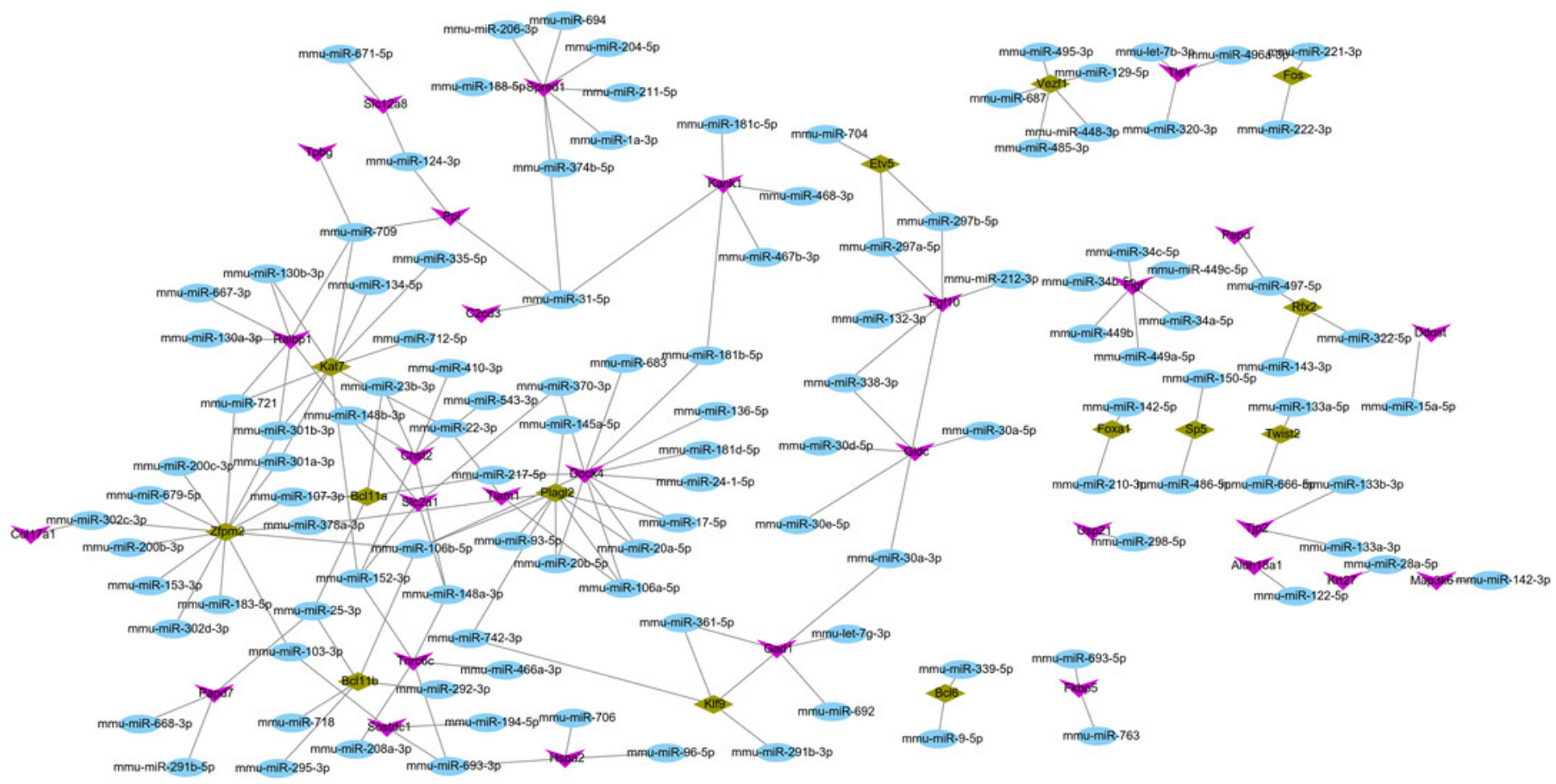




\section{Figure 6}

\section{The miRNA-mRNA regulatory network for the downregulated genes.}

\section{Blue: miRNAs; purple: downregulated genes; hazel-green: downregulated transcription}

\section{factors.}

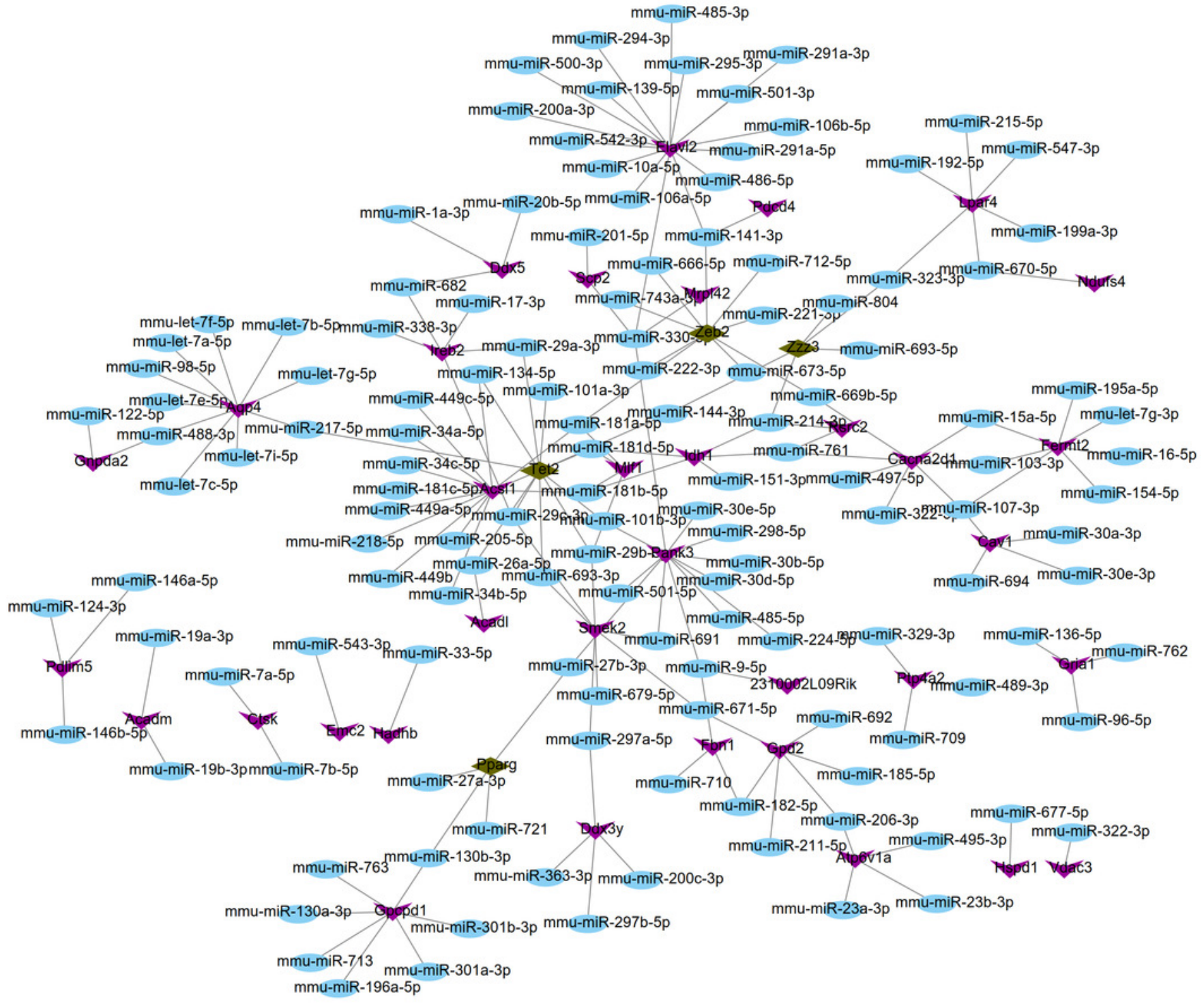




\section{Table $\mathbf{1}$ (on next page)}

KEGG pathways for differentially expressed genes in the PPI network 
1 Table 1 KEGG pathways for differentially expressed genes in the PPI network

\begin{tabular}{|c|c|c|c|}
\hline & Term & P-value & Genes \\
\hline \multirow[t]{25}{*}{ UP } & mmu04640:Hematopoietic & $1.89 \mathrm{E}-05$ & CD37, GP5, GP1BB, CD3E, ITGA5, CSF1, H2-EB1, \\
\hline & lineage & & ANPEP, ITGA3, ITGA2B... \\
\hline & mmu04512:ECM-receptor & $2.85 \mathrm{E}-05$ & VWF, LAMA1, CD47, LAMB3, GP5, GP1BB, \\
\hline & interaction & & ITGA5, ITGB4, ITGA3, ITGA2B... \\
\hline & mmu05164:Influenza A & 0.001774 & ICAM1, MYD88, HSPA2, SOCS3, IRF7, H2-EB1, \\
\hline & & & PML, H2-AB1, OAS2, CCL5... \\
\hline & mmu05168:Herpes & 0.002779 & FOS, MYD88, SOCS3, IRF7, H2-EB1, PML, PER2, \\
\hline & infection & & PER1, H2-AB1, OAS2... \\
\hline & mmu05150:Staphylococcus aureus & 0.005378 & ICAM1, SELP, C4B, H2-EB1, H2-AB1, SELPLG \\
\hline & infection & & \\
\hline & mmu04510:Focal adhesion & 0.007604 & VWF, LAMA1, LAMB3, ITGA5, RASGRF1, PAK4, \\
\hline & & & ITGB4, ITGA3, ZYX, MYL12A... \\
\hline & mmu05217:Basal cell carcinoma & 0.008061 & BMP4, WNT10A, WNT7B, WNT4, WNT3A, WNT6 \\
\hline & mmu05200:Pathways in cancer & 0.008918 & BMP4, WNT10A, RALBP1, WNT3A, PML, FGF10, \\
\hline & & & FOXO1, ITGA3, LAMA1, FOS... \\
\hline & mmu05323:Rheumatoid arthritis & 0.010803 & FOS, ICAM1, TNFSF13B, CSF1, H2-EB1, H2-AB1, \\
\hline & & & CCL5 \\
\hline & mmu04060:Cytokine-cytokine & 0.024171 & OSM, CXCL14, TNFSF13B, PRLR, CSF1, CXCR1, \\
\hline & receptor interaction & & CXCR2, CX3CL1, CCL5, BMP7... \\
\hline & mmu04151:PI3K-Akt & 0.029269 & EFNA1, CSF1, ITGB4, FGF10, ITGA3, CHAD, \\
\hline & pathway & & OSM, VWF, LAMA1, LAMB3... \\
\hline & mmu04611:Platelet activation & 0.02988 & VWF, ORAI1, GP5, GP1BB, PLCG2, MYL12A, \\
\hline & & & ITPR3, ITGA2B \\
\hline & mmu04514:Cell & 0.031608 & ICAM1, SIGLEC1, SELP, CLDN4, CLDN3, H2-EB1, \\
\hline & molecules (CAMs) & & H2-AB1, SELPLG, CLDN23 \\
\hline
\end{tabular}


mmu04668:TNF signaling pathway mmu04550:Signaling pathways regulating pluripotency of stem cells mmu05205:Proteoglycans cancer

Down mmu00190:Oxidative phosphorylation mmu05012:Parkinson's disease

mmu05016:Huntington's disease

mmu01100:Metabolic pathways

mmu05010:Alzheimer's disease

mmu04932:Non-alcoholic fatty liver disease (NAFLD)

mmu01200:Carbon metabolism

mmu01130:Biosynthesis

antibiotics

mmu00071:Fatty acid degradation

mmu00020:Citrate cycle (TCA cycle)

mmu00280:Valine, leucine and 5.07E-09
0.037976 FOS, ICAM1, SOCS3, CSF1, CX3CL1, CCL5, JUNB

0.038071 BMP4, WNT10A, WNT7B, WNT4, OTX1, WNT3A, WNT6, MEIS 1

in 0.042265 WNT10A, WNT7B, WNT4, TIAM1, ITGA5, WNT3A, PLCG2, ITPR3, WNT6, TWIST2

6.68E-28 UQCRC2, NDUFB3, ATP5E, NDUFB4, NDUFB5, NDUFB8, NDUFB9, COX7B, CYC1, NDUFB2...

1.48E-25 UQCRC2, NDUFB3, ATP5E，NDUFB4, NDUFB5, NDUFB8, NDUFB9, COX7B, CYC1, NDUFB2...

2.16E-25 UQCRC2, NDUFB3, POLR2G, ATP5E, NDUFB4, NDUFB5, NDUFB8, NDUFB9, PPARG, COX7B...

1.11E-23 UQCRC2, ATP5E, GNPDA2, CYC1, PDHB, CMBL, UQCR10, UQCR11, NDUFS4, IDH3G, MCEE...

8.60E-22 UQCRC2, NDUFB3, ATP5E, NDUFB4, NDUFB5, NDUFB8, NDUFB9, COX7B, CYC1, NDUFB2...

UQCRC2, NDUFB3, NDUFB4, NDUFB5, NDUFB8, NDUFB9, COX7B, CYC1, NDUFB2, UQCR10...

2.99E-11 ALDH6A1, ACADM, ACO2, SUCLG1, ECHS1, FBP2, ACAT2, PDHB, SDHB, TPI1...

of 2.59E-10 ACAA2, ACADM, ACO2, SUCLG1, ECHS1, AK2, FBP2, ACAT2, PDHB, CMBL...

1.21E-09 ECI1, ECI2, ACAA2, ACADSB, CPT2, ACADM, ECHS1, ACADL, ACAT2, HADHB...

SDHB，IDH3G，ACO2，SUCLG1，DLD，SDHD, IDH2, IDH1, PDHA1, FH1 ...

ACAA2, ALDH6A1, ACADSB, ACADM, ECHS1, 
isoleucine degradation

mmu01212:Fatty acid metabolism

mmu00640:Propanoate metabolism

pathway

mmu04146:Peroxisome

mmu00630:Glyoxylate

dicarboxylate metabolism

mmu01210:2-Oxocarboxylic

metabolism

mmu00620:Pyruvate metabolism

mmu00310:Lysine degradation
ACAT2, HADHB, DBT, MCEE, DLD...

2.57E-08 ACADVL, SCD1, ACAA2, ACADSB, ACSL1, ACADM, CPT2, ECHS1, ACADL, ACAT2...

2.58E-06 ALDH6A1，ACADM，SUCLG1，MCEE，ECHS1, ACAT2, PCCB, PCCA

muscle 1.47E-05 UQCRC2, UQCR10, CACNA2D1, UQCR11, COX8B, COX7A1, UQCRH, COX7B, CYC1, COX6B1...

signaling 2.07E-05 SCD1, ACSL1, ACADM, CPT2, PPARG, FABP3, AQP7, UCP1, ACADL, ACSL5...

1.66E-04 ECI2, ACSL1, ECH1, NUDT7, ABCD2, IDH2, IDH1, SCP2, SOD2, ACSL5

and $5.85 \mathrm{E}-04 \mathrm{ACO} 2, \mathrm{MCEE}, \mathrm{DLD}, \mathrm{ACAT} 2, \mathrm{PCCB}, \mathrm{PCCA}$

2 PPI, protein and protein interaction; KEGG, Kyoto encyclopedia of genes and genomes. 3 
Table 2 (on next page)

Hub genes in the protein-protein interaction network 
1 Table 2 Hub genes in the protein-protein interaction network

\begin{tabular}{|c|c|c|c|c|c|}
\hline Gene_Symbol & Degree & Gene_Symbol & Betweenness & Gene_Symbol & Closeness \\
\hline Uqcrc2 & 57 & Ehmt1 & 17581.75493 & Sod2 & 0.000195886 \\
\hline Sdhb & 55 & Fos & 16050.50737 & Eprs & 0.000194932 \\
\hline Atp5h & 53 & Icam 1 & 9986.745004 & Fos & 0.000194515 \\
\hline Ndufs3 & 52 & Sod2 & 9941.869934 & Icam1 & 0.000193573 \\
\hline Uqcr11 & 48 & Eprs & 9124.185173 & Pdk4 & 0.000192976 \\
\hline Ndufs2 & 48 & Pdk4 & 7466.1032 & Pparg & 0.000192827 \\
\hline Ehmt1 & 47 & Cav1 & 7458.835522 & Dld & 0.000192641 \\
\hline Dld & 47 & Helz2 & 7173.879054 & Aldh18a1 & 0.000192345 \\
\hline Cyc1 & 47 & Cops5 & 6791.408479 & Tpil & 0.000191939 \\
\hline Pmpcb & 46 & Dld & 6500.567441 & Foxo1 & 0.000191608 \\
\hline Ndufa9 & 46 & Gm128 & 6201.287809 & Ehmt1 & 0.000191168 \\
\hline Fos & 46 & Tpil & 6150.610424 & Socs3 & 0.000190949 \\
\hline Suclg1 & 45 & Pparg & 6092.823564 & Hspd1 & 0.000190767 \\
\hline Ndufv2 & 44 & Aldh18a1 & 5723.478434 & Aldh3a1 & 0.000190404 \\
\hline Ndufa8 & 44 & Tac1 & 5073.412043 & Aldh1a7 & 0.000190259 \\
\hline Ndufb5 & 43 & Gldc & 4915.109074 & Sirt3 & 0.00019015 \\
\hline Acadvl & 43 & Acadvl & 4870.244131 & Helz2 & 0.000190042 \\
\hline Uqcr10 & 42 & $\mathrm{Mb}$ & 4867.912647 & Ccng1 & 0.00018997 \\
\hline Ndufa5 & 42 & Bmp4 & 4710.157376 & Acadvl & 0.000189934 \\
\hline Uqcrh & 41 & Foxo1 & 4510.416535 & $\mathrm{Dbt}$ & 0.000189934 \\
\hline
\end{tabular}

\section{2}

3 
Table 3 (on next page)

Significant modules screened from protein-protein interaction network 
1

\begin{tabular}{|c|c|c|c|c|}
\hline Cluster & $\begin{array}{c}\text { Score } \\
\text { (Density*\#Nodes) }\end{array}$ & Nodes & Edges & Node IDs \\
\hline 1 & 15.692 & 39 & 612 & $\begin{array}{l}\text { Atp5j2, Ndufv2, Ndufs8, Ndufs2, Sdhb, Sdhd, } \\
\text { Ndufs4, Ndufs3, Ndufa8, Ndufc2, Uqcrc2, Cyc1, Etfb, } \\
\text { Atp5h, Atp5e, Uqcr10, Uqcr11, Pmpcb, Ndufb5, } \\
\text { Ndufb9, Ndufa9, Ndufa5, Suclg1, Ndufb8, Ndufb4, } \\
\text { Ndufb2, Uqcrh, Cox6c, Ndufc1, Ndufa1, } \\
\text { 1700029P11Rik, Ndufa7, Ndufa3, Ndufb3, Atp5f1, } \\
\text { Usmg5, Cycs, Cox6b1, Cox7b }\end{array}$ \\
\hline 2 & 6.241 & 58 & 362 & $\begin{array}{l}\text { Cpt2, Slc25a20, Ech1, Acadl, Acs11, Aldh1a7, } \\
\text { Aldh6a1, Aldh3a1, Acs15, Acat2, Acadsb, Eci1, } \\
\text { Echs1, Idh1, Dbt, Acadv1, Hadhb, Eci2, Acaa2, } \\
\text { Acadm, Etfdh, Pcca, Pccb, Etfa, Dld, Aco2, Idh3g, } \\
\text { Hspe1, Mrps15, Helz2, Irgm2, Rtp4, Mx2, Lgals3bp, } \\
\text { Figf, Pcyox11, Ola1, Ecm1, Sepp1, Serping1, Itih4, } \\
\text { Kng2, Kng1, Mrpl20, Mrps28, Ptcd3, Mrpl30, } \\
\text { Mrp142, Mrp153, Mrp19, Mrp127, Mrp112, Dhx58, } \\
\text { Oas12, Oas2, Oas11, Irf7, Fh1 }\end{array}$ \\
\hline
\end{tabular}




\section{Table 4 (on next page)}

KEGG pathways for differentially expressed genes in module analysis 


\begin{tabular}{|c|c|c|c|}
\hline Module & Term & P-value & Genes \\
\hline \multirow[t]{8}{*}{1} & $\begin{array}{l}\text { mmu00190:Oxidative } \\
\text { phosphorylation }\end{array}$ & $1.80 \mathrm{E}-55$ & $\begin{array}{l}\text { UQCRC2, NDUFB3, ATP5E, NDUFB4, NDUFB5, } \\
\text { NDUFB8, NDUFB9, COX7B, CYC1, NDUFB2... }\end{array}$ \\
\hline & $\begin{array}{l}\text { mmu05012:Parkinson's } \\
\text { disease }\end{array}$ & $2.20 \mathrm{E}-54$ & $\begin{array}{l}\text { UQCRC2, NDUFB3, ATP5E, NDUFB4, NDUFB5, } \\
\text { NDUFB8, NDUFB9, COX7B, CYC1, NDUFB2... }\end{array}$ \\
\hline & $\begin{array}{l}\text { mmu05010:Alzheimer's } \\
\text { disease }\end{array}$ & $9.89 \mathrm{E}-52$ & $\begin{array}{l}\text { UQCRC2, NDUFB3, ATP5E, NDUFB4, NDUFB5, } \\
\text { NDUFB8, NDUFB9, COX7B, CYC1, NDUFB2... }\end{array}$ \\
\hline & $\begin{array}{l}\text { mmu05016:Huntington's } \\
\text { disease }\end{array}$ & 4.97E-50 & $\begin{array}{l}\text { UQCRC2, NDUFB3, ATP5E, NDUFB4, NDUFB5, } \\
\text { NDUFB8, NDUFB9, COX7B, CYC1, NDUFB2... }\end{array}$ \\
\hline & $\begin{array}{l}\text { mmu04932:Non-alcoholic } \\
\text { fatty liver disease (NAFLD) }\end{array}$ & $1.49 \mathrm{E}-45$ & $\begin{array}{l}\text { UQCRC2, NDUFB3, NDUFB4, NDUFB5, NDUFB8, } \\
\text { NDUFB9, COX7B, CYC1, NDUFB2, UQCR10... }\end{array}$ \\
\hline & $\begin{array}{l}\text { mmu01100:Metabolic } \\
\text { pathways }\end{array}$ & $1.92 \mathrm{E}-27$ & $\begin{array}{l}\text { UQCRC2, NDUFB3, ATP5E, NDUFB4, NDUFB5, } \\
\text { NDUFB8, NDUFB9, COX7B, CYC1, NDUFB2... }\end{array}$ \\
\hline & $\begin{array}{l}\text { mmu04260:Cardiac muscle } \\
\text { contraction }\end{array}$ & $3.23 \mathrm{E}-08$ & $\begin{array}{l}\text { UQCRC2, UQCR10, UQCR11, UQCRH, COX7B, } \\
\text { CYC1, COX6B1, COX6C }\end{array}$ \\
\hline & $\begin{array}{l}\text { mmu00020: Citrate cycle } \\
\text { (TCA cycle) }\end{array}$ & 0.008597 & SDHB, SUCLG1, SDHD \\
\hline \multirow[t]{5}{*}{2} & $\begin{array}{l}\text { mmu00071:Fatty } \\
\text { degradation }\end{array}$ & $1.63 \mathrm{E}-18$ & $\begin{array}{l}\text { ECI1, ECI2, ACAA2, ACADSB, CPT2, ACADM, } \\
\text { ECHS1, ACADL, ACAT2, HADHB... }\end{array}$ \\
\hline & $\begin{array}{l}\text { mmu01212:Fatty acid } \\
\text { metabolism }\end{array}$ & $1.89 \mathrm{E}-14$ & $\begin{array}{l}\text { ACADVL, ACAA2, ACADSB, ACSL1, ACADM, } \\
\text { CPT2, ECHS1, ACADL, ACAT2, ACSL5... }\end{array}$ \\
\hline & $\begin{array}{l}\text { mmu00280:Valine, leucine } \\
\text { and isoleucine degradation }\end{array}$ & 4.29E-14 & $\begin{array}{l}\text { ACAA2, ALDH6A1, DBT, ACADSB, ACADM, DLD, } \\
\text { ECHS1, ACAT2, PCCB, PCCA... }\end{array}$ \\
\hline & $\begin{array}{l}\text { mmu01200:Carbon } \\
\text { metabolism }\end{array}$ & $9.82 \mathrm{E}-11$ & $\begin{array}{l}\text { ALDH6A1, ACADM, IDH3G, ACO2, DLD, ECHS1, } \\
\text { IDH1, FH1, ACAT2, PCCB, PCCA }\end{array}$ \\
\hline & mmu01130:Biosynthesis of & $1.52 \mathrm{E}-10$ & ACAA2, ACADM, ACO2, ECHS1, ACAT2, HADHB, \\
\hline
\end{tabular}


antibiotics

mmu00640:Propanoate

metabolism

mmu01100:Metabolic

pathways

mmu00630:Glyoxylate and

9.74E-06

dicarboxylate metabolism

mmu00020:Citrate

cycle $1.46 \mathrm{E}-05$

(TCA cycle)

mmu00410:beta-Alanine

$5.01 \mathrm{E}-04$

ALDH6A1, ACADM, ECHS1, ALDH3A1

metabolism

mmu03320:PPAR signaling

$5.45 \mathrm{E}-04$

ACSL1, ACADM, CPT2, ACADL, ACSL5

pathway

mmu03010:Ribosome

5.86E-04 MRPL12, MRPS15, MRPL27, MRPL9, MRPL30, MRPL20

mmu04146:Peroxisome

6.26E-04 ECI2, ACSL1, ECH1, IDH1, ACSL5

mmu01210:2-Oxocarboxylic

0.003631

acid metabolism

mmu00062:Fatty

acid 0.006757 ACAA2, ECHS1, HADHB

elongation

mmu00620:Pyruvate

0.014816 DLD, FH1, ACAT2

metabolism 


\section{Table 5 (on next page)}

Small molecule drugs predicted by Cmap database 
1 Table 5 Small molecule drugs predicted by Cmap database

\begin{tabular}{lllll}
\hline Cmap name & Mean & $\mathrm{N}$ & Enrichment & $\mathrm{P}$ \\
adiphenine & -0.727 & 5 & -0.958 & 0 \\
DL-PPMP & -0.672 & 1 & -0.95 & --- \\
decitabine & -0.617 & 1 & -0.915 & --- \\
topiramate & -0.596 & 1 & -0.899 & --- \\
5186324 & -0.578 & 1 & -0.886 & --- \\
5213008 & -0.573 & 1 & -0.883 & --- \\
sulindac sulfide & -0.56 & 1 & -0.869 & --- \\
BW-B70C & -0.555 & 1 & -0.865 & --- \\
5186223 & -0.553 & 1 & -0.863 & --- \\
isoxicam & -0.665 & 5 & -0.852 & 0.00016 \\
cefamandole & -0.574 & 4 & -0.845 & 0.00105 \\
isoflupredone & -0.623 & 3 & -0.815 & 0.01256 \\
5140203 & -0.5 & 1 & -0.815 & --- \\
tyrphostin AG-1478 & -0.497 & 1 & -0.812 & --- \\
12,13-EODE & -0.497 & 1 & -0.812 & --- \\
STOCK1N-35696 & -0.582 & 2 & -0.808 & 0.07269 \\
lisuride & -0.57 & 5 & -0.808 & 0.00058 \\
5149715 & -0.483 & 1 & -0.801 & --- \\
Prestwick-692 & -0.629 & 4 & -0.799 & 0.0032 \\
carbimazole & -0.568 & 3 & -0.792 & 0.01831 \\
PF-00539745-00 & -0.517 & 3 & -0.79 & 0.01893 \\
Prestwick-691 & -0.562 & 3 & -0.78 & 0.02195 \\
indoprofen & -0.519 & 4 & -0.779 & 0.00493 \\
5162773 & -0.457 & 1 & -0.778 & --- \\
iloprost & -0.494 & 3 & -0.759 & 0.02876 \\
5151277 & -0.427 & 1 & -0.755 & --- \\
splitomicin & -0.423 & 1 & -0.752 & --- \\
Prestwick-1082 & -0.625 & 3 & -0.743 & 0.03481 \\
clorsulon & -0.567 & 4 & -0.739 & 0.00915 \\
vigabatrin & -0.539 & 3 & -0.738 & 0.03684 \\
3-acetamidocoumarin & -0.538 & 4 & -0.736 & 0.00959 \\
thiamphenicol & -0.541 & 5 & -0.734 & 0.00274 \\
levobunolol & -0.48 & 4 & -0.731 & 0.01056 \\
oxolamine & -0.551 & 4 & -0.72 & 0.01241 \\
cinchonine & -0.494 & 4 & -0.716 & 0.01327 \\
trimethobenzamide & -0.536 & 5 & -0.714 & 0.00415 \\
atracurium besilate & -0.559 & 3 & -0.712 & 0.04853 \\
levomepromazine & -0.517 & 4 & -0.71 & 0.01456 \\
chloropyrazine & -0.492 & 4 & -0.708 & 0.0151 \\
\hline & & & &
\end{tabular}




\begin{tabular}{|c|c|c|c|c|}
\hline tranexamic acid & -0.548 & 5 & -0.703 & 0.00505 \\
\hline isometheptene & -0.517 & 4 & -0.701 & 0.01657 \\
\hline benzbromarone & -0.492 & 3 & -0.698 & 0.05574 \\
\hline heptaminol & -0.544 & 5 & -0.687 & 0.00685 \\
\hline trihexyphenidyl & -0.406 & 3 & -0.68 & 0.06572 \\
\hline Prestwick-642 & -0.4 & 4 & -0.68 & 0.02304 \\
\hline viomycin & -0.53 & 4 & -0.678 & 0.02383 \\
\hline naringenin & -0.463 & 4 & -0.663 & 0.0291 \\
\hline colistin & -0.415 & 4 & -0.655 & 0.03288 \\
\hline guanabenz & -0.488 & 5 & -0.649 & 0.0131 \\
\hline canadine & -0.442 & 4 & -0.647 & 0.0369 \\
\hline sulmazole & -0.442 & 3 & -0.646 & 0.08926 \\
\hline Gly-His-Lys & -0.512 & 3 & -0.642 & 0.09278 \\
\hline terazosin & -0.444 & 4 & -0.637 & 0.04176 \\
\hline sulfadimethoxine & -0.501 & 5 & -0.628 & 0.01852 \\
\hline iopamidol & -0.411 & 4 & -0.622 & 0.05101 \\
\hline PHA-00745360 & -0.432 & 8 & -0.62 & 0.00172 \\
\hline iodixanol & -0.423 & 3 & -0.619 & 0.11656 \\
\hline ribavirin & -0.469 & 4 & -0.614 & 0.05616 \\
\hline atractyloside & -0.485 & 5 & -0.593 & 0.03218 \\
\hline mycophenolic acid & -0.42 & 3 & -0.591 & 0.15331 \\
\hline Prestwick-1103 & -0.472 & 4 & -0.59 & 0.07607 \\
\hline aciclovir & -0.415 & 6 & -0.587 & 0.01712 \\
\hline triflupromazine & -0.446 & 4 & -0.578 & 0.08749 \\
\hline rifampicin & -0.415 & 4 & -0.577 & 0.08825 \\
\hline acemetacin & -0.477 & 4 & -0.571 & 0.09443 \\
\hline bumetanide & -0.459 & 4 & -0.57 & 0.09533 \\
\hline josamycin & -0.407 & 5 & -0.551 & 0.05727 \\
\hline trapidil & -0.404 & 3 & -0.544 & 0.23612 \\
\hline proguanil & -0.463 & 3 & -0.524 & 0.27656 \\
\hline
\end{tabular}

